
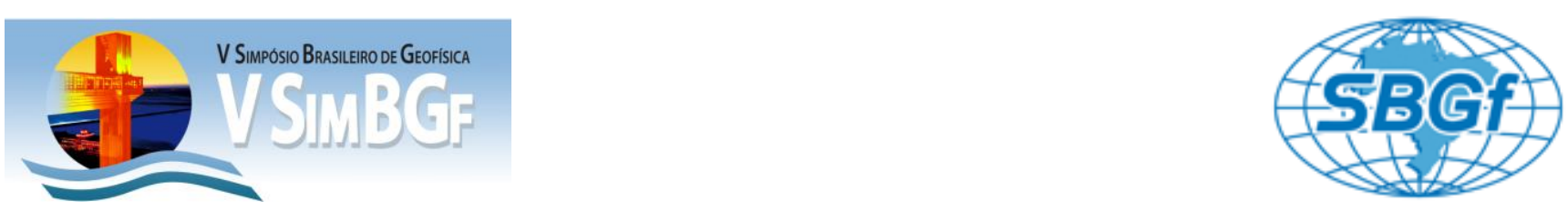

\title{
Estudo gravimétrico do limite Cráton São Francisco - Faixa Araçuaí na região de Grão Mogol, Minas Gerais
}

Guilherme F. da Silva *1, Deusavan Sales Filho ${ }^{1}$, Mônica G. Von Huelsen ${ }^{2}$, Amanda Almeida Rocha ${ }^{1}$, Sidinei Sebastião Tomás $^{2}$, José O. de Araújo Filho ${ }^{1}$, Hortência Sousa Lamblém ${ }^{1}$

1 - Instituto de Geociências, Universidade de Brasília

2 - Observatório Sismológico, Universidade de Brasília
Arial normal, 9. Numero de palavras: cerca de 300.: Fonte: Arial normal, 9. Numero de palavras: cerca de 300.: Fonte: Arial normal, 9. Fonte: Arial normal, 9. Numero de palavras: cerca de 300.:

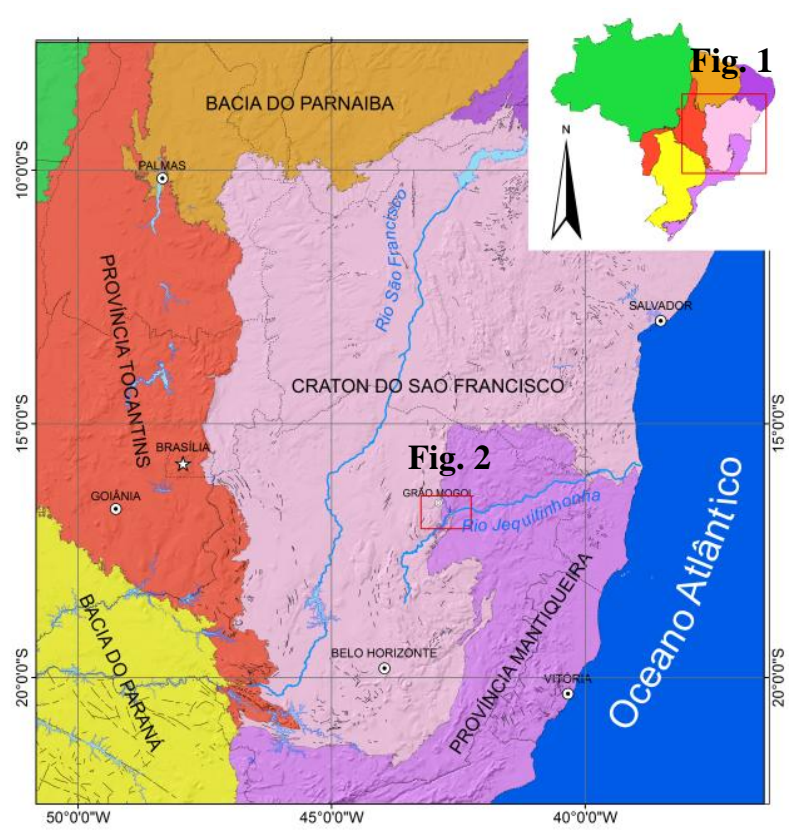

Figura 1: Contexto tectônico da área de estudo, destacada em vermelho (modificado de Bizzi, et al. 2003)

Fonte: Arial normal, 9. Numero de palavras: cerca de 300. 300: Fonte: Arial normal, 9. Numero de palavras: cerca de 300.: Fonte: Arial normal, 9. Numero de palavras: cerca de 300.: Fonte: Arial normal, 9. Numero 


\section{Mapa Geológico do Entorno da UHE - Irapé}

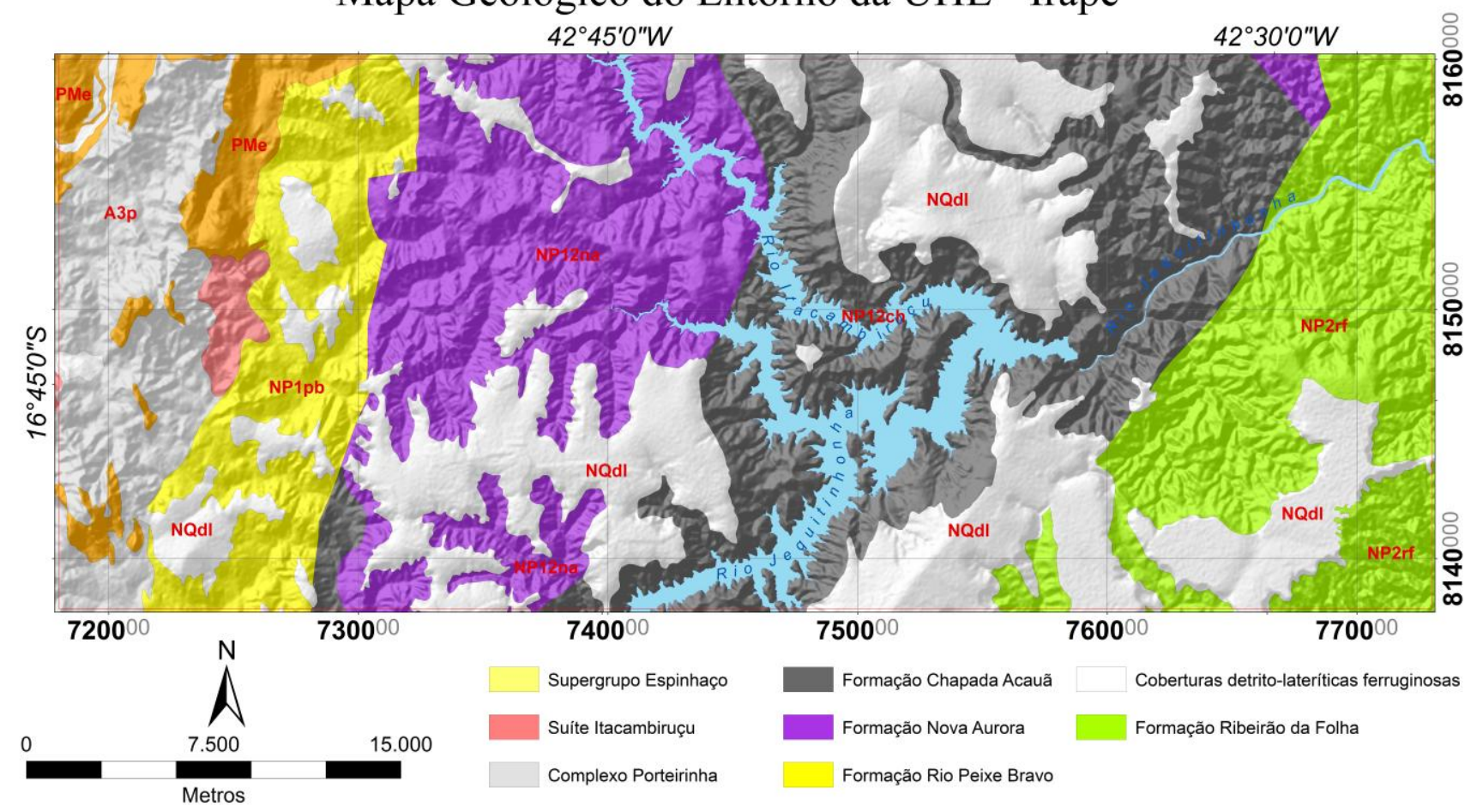

Figura 2: Mapa Geológico do Entorno da UHE Irapé fusionado com imagem de relevo sombreado da SRTM (modificado de Araújo Filho et al., 2010)

\section{Resultados}

Fonte: Arial normal, 9. Numero de palavras: cerca de 500-600.: Fonte: Arial normal, 9. Numero de palavras: cerca de 500-600. 500-600: Fonte: Arial normal, 9. Numero de palavras: cerca de 500-600.: Fonte: Arial normal, 9. Numero de palavras: cerca de 500-600.: Fonte: Arial normal, 9. Numero de palavras: cerca de 500-600.: Fonte: Arial normal, 9. Numero de palavras: cerca de 500-600.: Fonte: Arial normal, 9. Numero de palavras: cerca de 500-600.: Fonte: Arial normal, 9. Numero de palavras: cerca de 500-600.: Fonte: Arial palavras: cerca de 500-600.: Fonte: Arial normal, 9. Numero de palavras: cerca de 500-600.: Fonte: Arial normal, 9. Fonte: Arial normal, 9. Numero de palavras: cerca de 500-600.: Fonte: Arial normal, 9. Numero de palavras: cerca de 500-600. 500-600: Fonte: Arial normal, 9. Numero de palavras: cerca de 500-600.: Fonte: Arial normal, 9. Numero de palavras: cerca de 500-600.: Fonte: Arial normal, 9. Numero de palavras: cerca de 500-600.: Fonte: Arial normal, 9. Numero de palavras: cerca de 500-600.: Fonte: Arial normal, 9. Numero de palavras: cerca de 500-600.: Fonte: Arial normal, 9. Numero de palavras: cerca de 500-600.: Fonte: Arial normal, 9. Numero de palavras: cerca de 500-600.: Fonte: Arial normal, 9. Numero de palavras: cerca de 500-600.: Fonte: Arial normal, 9. Fonte: Arial normal, 9. Numero de palavras: cerca de 500-600.: Fonte: Arial normal, 9. Numero de palavras: cerca de 500-600. 500600: Fonte: Arial normal, 9. Numero de palavras: cerca de 500-600.: Fonte: Arial normal, 9. Numero de palavras: cerca de 500-600.: Fonte: Arial normal, 9. Numero de palavras: cerca de 500-600.: Fonte: Arial normal, 9. Numero de palavras: cerca de 500-600.: Fonte: Arial normal, 9. Numero de palavras: cerca de 500-600.: Fonte: Arial normal, 9. Numero de palavras: cerca de 500-600.: Fonte: Arial normal, 9. normal, 9. Numero de palavras: cerca de 500-600.: Fonte: Arial normal, 9. Numero de palavras: cerca de 500-600.: Fonte: Arial normal, 9. Numero de palavras: cerca de 500-600.: Fonte: Arial normal, 9. Fonte: Arial normal, 9. Numero de palavras: cerca de 500-600.: Fonte: Arial normal, 9. Numero de palavras: cerca de 500-600. 500-600: Fonte: Arial normal, 9. Numero de palavras: cerca de 500-600.: Fonte: Arial normal, 9. Numero de palavras: cerca de 500-600.: Fonte: Arial normal, 9. Numero de palavras: cerca de 500-600.: Fonte: Arial normal, 9. Numero de palavras: cerca de 500-600.: Fonte: Arial normal, 9. Numero de palavras: cerca de 500-600.: Fonte: Arial normal, 9. Numero de palavras: cerca de 500-600.: Fonte: Arial normal, 9. normal, 9. Numero de palavras: cerca de 500-600.: Fonte: Arial normal, 9. Numero de palavras: cerca de 500-600.: Fonte: Arial normal, 9. Numero de palavras: cerca de 500-600.: Fonte: Arial normal, 9. Fonte: Arial normal, 9. Numero de palavras: cerca de 500-600.: Fonte: Arial normal, 9. Numero de palavras: cerca de 500-600. 500-600: Fonte: Arial normal, 9. Numero de palavras: cerca de 500-600.: Fonte: Arial normal, 9. Numero de palavras: cerca de 500-600. Fonte: Arial normal, 9. Numero de palavras: cerca de 


\section{Discussão e Conclusões (Arial Bold, 9)}

Fonte: Arial normal, 9. Numero de palavras: cerca de 300.: Fonte: Arial normal, 9. Numero de palavras: cerca de 300. 300: Fonte: Arial normal, 9. Numero de palavras: cerca de 300.: Fonte: Arial normal, 9. Numero de palavras: cerca de 300.: Fonte: Arial normal, 9. Numero de palavras: cerca de 300.: Fonte: Arial normal, 9. Numero de palavras: cerca de 300.: Fonte: Arial normal, 9. Numero de palavras: cerca de 300.: Fonte: Arial normal, 9. Numero de palavras: cerca de 300.: Fonte: Arial normal, 9. Numero de palavras: cerca de 300.: Fonte: Arial normal, 9. Numero de palavras: cerca de 300.: Fonte: Arial normal, 9. Fonte: Arial normal, 9. Numero de palavras: cerca de 300.: Fonte: Arial normal, 9. Numero de palavras: cerca de 300. 300: Fonte: Arial normal, 9. Numero de palavras: cerca de 300.: Fonte: Arial normal, 9. Numero de palavras: cerca de 300.: Fonte: Arial normal, 9. Numero de palavras: cerca de 300.: Fonte: Arial normal, 9. Numero de palavras: cerca de 300.: Fonte: Arial normal, 9. Numero de palavras: cerca de 300.: Fonte: Arial normal, 9. Numero de palavras: cerca de 300.: Fonte: Arial normal, 9. Numero de palavras: cerca de 300.: Fonte: Arial normal, 9. Numero de palavras: cerca de 300.: Fonte: Arial normal, 9. Fonte: Arial normal, 9. Numero de palavras: cerca de 300.: Fonte: Arial normal, 9. Numero de palavras: cerca de 300. 300: Fonte: Arial normal, 9. Numero de palavras: cerca de 300.: Fonte: Arial normal, 9. Numero de palavras: cerca de 300.: Fonte: Arial normal, 9. Numero de palavras: cerca de 300.: Fonte: Arial normal, 9. Numero de palavras: cerca de 300.: Fonte: Arial normal, 9. Numero de palavras: cerca de 300.: Fonte: Arial normal, 9. Numero de palavras: cerca de 300.: Fonte: Arial normal, 9. Numero de palavras: cerca de 300.: Fonte: Arial normal, 9. Numero de palavras: cerca de 300.: Fonte: Arial normal, 9. Fonte: Arial normal, 9. Numero de palavras: cerca de 300.: Fonte: Arial normal, 9. Numero de palavras: cerca de 300. 300: Fonte: Arial normal, 9. Numero de palavras: cerca de 300.: Fonte: Arial normal, 9. Numero de palavras: cerca de 300.: Fonte: Arial normal, 9.
Numero de palavras: cerca de 400.: Fonte: Arial normal, 9. Numero de palavras: cerca de 400.: Fonte: Arial normal, 9. Numero de palavras: cerca de 400.: Fonte: Arial normal, 9. Numero de palavras: cerca de 400: Fonte: Arial normal, 9.

\section{Agradecimentos}

Os autores agradecem ao Conselho Nacional de Desenvolvimento Científico e Tecnológico - CNPq (pelo auxílio financeiro e aprovação do projeto de pesquisa) e ao Observatório Sismológico da Universidade de Brasília pelo suporte e logística para as atividades de campo.

\section{Referências (Arial Bold, 9) Veja normas da RBGf}

Almeida, F. F. M. de, 1969. Diferenciação tectônica da Plataforma Brasileira (Tectonic differentiation of the Brazilian Platform). Proceedings 33rd. Brazilian Geological Congress, Salvador(BA), Brazil, vol. 1: 29-46.

Arora et al. (06 co-authors), 1999. 2D geoelectrical model for the Parnaíba conductivity anomaly, north-northeast Brazil and its tectonic implications. Tectonophysics, 302: 57-69.

Bott, M. H. P., 1982. Stress based tectonic mechanisms at passive continental margins. In: Scrutton, R. A. (Ed.), Dynamics of Passive Margins. AGU Geodynamic Series, Washington (DC), USA, vol. 6: 147-153.

Chandler, V. W., Kiski, H. S., Hinze, W. J. \& Braile, L. W., 1981. Analysis of multisource gravity and magnetic anomaly data sets by moving-window applications of Poisson's theorem. Geophysics, 46: 30-39.

Figueiredo, M. C. H., 1980. Geochemistry of high-grade metamorphic rocks, Northeastern Bahia, Brazil. Ph. D. Thesis, Department of Geology of the University of Western Ontario, Canada.

Turner, F. J. \& Werhoogen, J., 1960. Igneous and metamorphic petrology. 2nd ed., McGraw Hill, New York, USA.

figura 
figura

Figura 4 - Qqwertqwertqwertqwertqwertqwertqwertqwertqwertqwertqwertqwertqwertqwertqwert. 